\title{
A case of an atraumatic implant failure after primary rotating-hinge total knee arthroplasty
}

\author{
Lucas Petitqueux, Karen Verhulst, Jan Dauwe, Dirk Dauwe
}

From the AZ Delta Hospital, Belgium

\begin{abstract}
Rotating-hinge knee implants are fully constrained knee prostheses commonly used for revision total knee arthroplasty. Nevertheless, rotating-hinge devices have been increasingly utilized in primary setting. Complications are inevitable in orthopedic surgery, however, implant breakage after RHK arthroplasty has been rarely described in medical literature.

We present a rare case of 70-year-old Caucasian, male patient who suffered an atraumatic femoral stem breakage in a primary NexGen ${ }^{\circledR}$ Rotating Hinge Knee (Zimmer-Biomet $^{\circledR}$, Warsaw, IN, USA).
\end{abstract}

Keywords : Rotating-hinge knee; total knee arthroplasty ; mechanical failure ; implant breakage ; implant failure; femoral stem breakage.

\section{INTRODUCTION}

Rotating-hinge knee [RHK] implants are fully constrained knee prostheses commonly used for revision total knee arthroplasty [TKA]. However, rotating-hinge devices have been increasingly utilized in primary setting (1). Indications for primary rotating-hinge knee arthroplasty are limited but have been thoroughly described in medical literature (2). RHK implants provide extreme joint stability and are therefore preferably indicated in older patients with advanced axial deformities, severe bone defect and complex knee instability (2). Despite the rather notorious history of unacceptably high failure rates

Informed consent. A written informed consent was obtained from the patient. and complications (3), good clinical results have been reported by several authors using modern third generation rotating hinges $(4,5)$. However, other studies present contradictory findings and evidence is lacking on long-term follow-up (6). Complications are inevitable in orthopedic surgery and have been abundantly described from infection and loosening up to periprosthetic fractures and mechanical failure. Failure of rotating-hinge knee arthroplasties specifically has been described before such as Yoke fractures or the disengagement of the hinge-post extension $(7,8)$.

We present a case of an atraumatic implant breakage in a 70-year-old male patient five years after primary rotating-hinge total knee arthroplasty. To the best of our knowledge, this is the first case of this specific kind a femoral stem breakage in a

\footnotetext{
Lucas Petitqueux ${ }^{1,2}$,

Karen Verhulst ${ }^{3}$,

- Jan Dauwe ${ }^{4}$,

- Dirk Dauwe ${ }^{2}$

${ }^{1}$ Catholic University of Leuven, Belgium.

${ }^{2}$ Department of Orthopaedic and Trauma Surgery, AZ Delta, Belgium.

${ }^{3}$ Department of Ophthalmology, AZ Delta, Belgium.

${ }^{4}$ Department of Orthopaedic Surgery, University Hospitals Leuven, Belgium.

Correspondence : Dr Jan Dauwe, University Hospitals Leuven, Herestraat 49, 3000 Leuven.

Email : jan.dauwe@kuleuven.be

- 2021, Acta Orthopædica Belgica.
} 
primary NexGen Rotating Hinge Knee (ZimmerBiomet $^{\circledR}$, Warsaw, IN, USA).

\section{CASE REPORT}

A 70-year-old male Caucasian patient was treated at the orthopedics department with a primary total knee arthroplasty [TKA] because of terminal tricompartimental gonarthritis of the knee. A NexGen Rotating Hinge Knee (ZimmerBiomet $^{\circledR}$, Warsaw, IN, USA) was implanted since severe mediolateral instability was documented. Postoperatively and after rehabilitation, no complications were noted and the patient was very satisfied with the result. His discomfort was solved and he achieved a good knee function with a full range of motion expected from a RHK prosthesis.

Five years after initial TKA, the patient presented with acute atraumatic pain and swelling in the knee. The patient encountered regular episodes of discomfort and swelling in the knee for over two years. An arthrocentesis of the knee was performed. There were no signs of infection, cultures and antibiogram were negative, no elevated CRP [C-reactive protein] levels were found in blood samples. Control X-ray showed no abnormalities. A Technecium-99m bone scintigraphy and a low dose SPECT-CT were performed. Signs of a small fracture line were observed on the medial side of the distal fourth of femoral component of the total knee implant. An active spot anterior against the implant in the medial condyle of the femur was reported. There were no signs of loosening of the prosthesis. The patient was followed-up (conservatively) with planned visits at the department of orthopedic surgery. Wait and see policy was installed and adequate pain medication was prescribed.

A second arthrocentesis was performed (because of intermittent knee swelling), 8 months after the first, the cytologic examination of the synovium fluid revealed the presence of black pigment (foreign body), still no signs of infection were documented.

Seven years after the primary rotating hinge knee [RHK] arthroplasty, another X-ray was obtained because of worsening of the patient's complaints. He experienced more pain and had trouble walking. This X-ray image revealed a fracture of the RHK

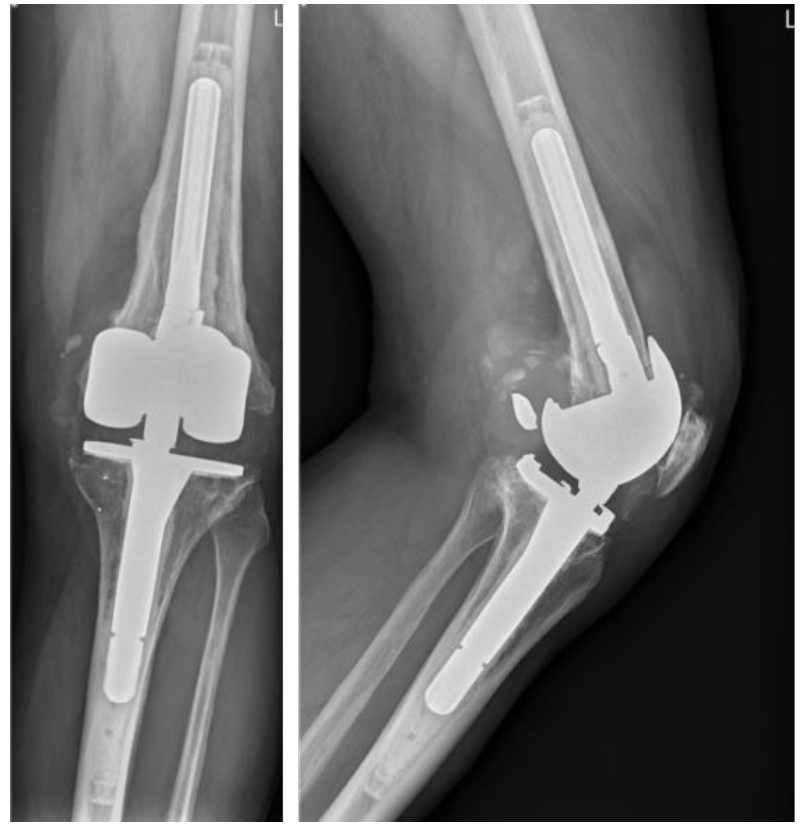

Figure 1. - Anteroposterior and lateral X-ray of the left knee showing implant breakage.

implant on the transition of the femoral stem and the femoral component of the hinge. Broken parts of the implant were visible outside of the joint (Fig 1). A technecium- $99 \mathrm{~m}$ bone scintigraphy and a low dose SPECT-CT were performed for the second time. Healing of the fracture of the left medial cortical femur was visualized. Loosening of the femoral component of the implant was observed. The active spot anterior against the implant in the medial condyle of the femur was still lighting up. Because of these findings, a revision RHK prosthesis was implanted under spinal anesthesia two days later. Follow-up one month after the operation, the patient was very satisfied and a good range of motion of the knee was noted.

\section{DISCUSSION}

The aim of this report was to present a rare case of an atraumatic implant failure after primary rotating hinge knee [RHK] arthroplasty. RHK implants are commonly used in revision settings where there is a serious need for component constraint, however, they are increasingly used in primary setting (2). In a cohort study, Baker et al. (1) reported that the 


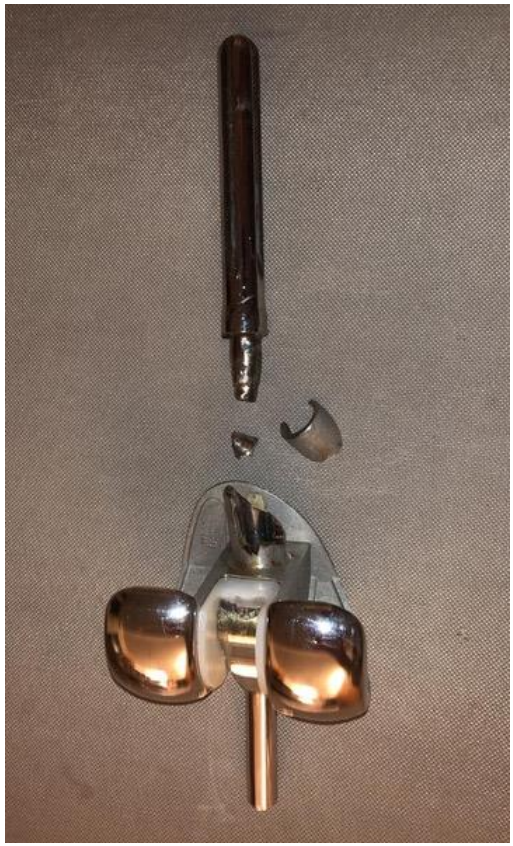

Figure 2. - Image of the broken implant viewed from posterior.

survival rate of osteo-arthritis patients who underwent primary RHK surgery was $96.8 \%$ after five years. Infection, peri-prosthetic fractures and aseptic loosening were the most frequent indications requiring revision surgery. Cases of revision due to a breakage of the RHK implant are very scarce. Gilg et al. (9) demonstrated that the incidence of knee implant fracture after knee arthroplasty is $0.13-0.3 \%$. Freisenbichler et $\mathrm{al}^{7}$ reported four cases of Yoke fractures in RHK implants. The fractures occurred on the hinged tibial insert at the transition zone from the conical to the cylindrical part. In their paper, they hypothesized that the high axial load during flexion with millimeters of displacement and anterior load caused the breakage. Sandiford et al. (10) reported three cases of RHK implants who all failed at the stem condylar junction. Debdut et al. (8) reported a single case of disengagement of the post-hinge extension because of loosening of the locking mechanism due to internal rotation of the femoral component. All the cases showed an atraumatic aseptic breakage of an RHK arthroplasty due to loosening of a component and the high axial loads. In the current case report, we describeb an atraumatic RHK implant failure at the transition of the femoral stem and the femoral component of the hinge prosthesis (Fig 2). In our opinion a plausible explanation for the failure of this specific case could be that the femoral component of the implant loosened after five years. Therefore, loading stresses on the femoral component changed and the implant broke on a weak point being the transition of the femoral stem and the femoral component of the hinge.

\section{REFERENCES}

1. Baker P, Critchley R, Gray A, Jameson S, Gregg P, Port A. et al. Mid-term survival following primary hinged total knee replacement is good irrespective of the indication for surgery. Knee Surg Sport Traumatol Arthrosc. 2014 ; 22(3) : 599-608.

2. Dauwe J, Vandenneucker H. Indications for primary rotating-hinge total knee arthroplasty. Is there consensus? Acta Orthop Belg. 2018 ; 84(3) : 245-250

3. James V. Bono M. Revision Total Knee Arthroplasty. $2005: 270-273$.

4. Jones RE, Barrack RL, Skedros J. Modular, mobilebearing hinge total knee arthroplasty. Clin Orthop Relat Res. 2001 ; (392) : 306-14.

5. Barrack RL. Evolution of the rotating hinge for complex total knee arthroplasty. Clin Orthop Relat Res. 2001 ; (392) : 292-9.

6. Martin JR, Beahrs TR, Stuhlman CR, Trousdale RT. Complex Primary Total Knee Arthroplasty. J Bone Jt Surg. 2016 ; 98(17) : 1459-70.

7. Friesenbichler J, Schwarzkopf R, Sadoghi P, et al. Failure rate of a rotating hinge knee design due to yoke fracture of the hinged tibial insert : a retrospective data analysis and review of the literature. Int Orthop. 2012 ; 36(5) : 993-998.

8. Biswas D, Haughom B, Mayle RE, Della Valle CJ. Case report: Failure of rotating-hinge total knee prosthesis by disengagement of the hinge-post extension knee. Clin Orthop Relat Res. 2013 ; 471(4) : 1389-92.

9. Gilg MM, Zeller CW, Leitner L, Leithner A, Labek G, Sadoghi P. The incidence of implant fractures after knee arthroplasty. Vol. 24, Knee Surg, Sports Traumatol, Arthrosc. 2016 : 3272-9.

10. Sandiford NA, Phillips JR, Back DL, Toms AD. Three Cases of Femoral Stem Failure in Rotating Hinge Revision Total Knee Arthroplasty: Causes and Surgical Considerations. Clin Orthop Surg. 2018 ; 10(2) : 260-4. 